\title{
Visualization of Mappings between the Gene Ontology and Cluster Trees
}

\author{
Ilir Jusufi ${ }^{a}$, Andreas Kerren $^{a *}$, Vladyslav Aleksakhin $^{a}$, and Falk Schreiber ${ }^{b}$ \\ ${ }^{a}$ Linnaeus University, School of Computer Science, Physics and Mathematics, Växjö, Sweden; \\ ${ }^{b}$ IPK Gatersleben and Martin-Luther University Halle-Wittenberg, Germany
}

\begin{abstract}
Ontologies and hierarchical clustering are both important tools in biology and medicine to study high-throughput data such as transcriptomics and metabolomics data. Enrichment of ontology terms in the data is used to identify statistically overrepresented ontology terms, giving insight into relevant biological processes or functional modules. Hierarchical clustering is a standard method to analyze and visualize data to find relatively homogeneous clusters of experimental data points. Both methods support the analysis of the same data set, but are usually considered independently. However, often a combined view is desired: visualizing a large data set in the context of an ontology under consideration of a clustering of the data. This paper proposes a new visualization method for this task.
\end{abstract}

Keywords: Gene Ontology, hierarchical clustering, visualization, mappings

\section{INTRODUCTION}

Ontologies play an important role in biology and medicine to structure biological knowledge. An ontology is a set of controlled, relational vocabularies of terms commonly used in particular areas of science. Ontologies are used to structure and standardize biological knowledge to support data integration and information exchange. Examples are Gene Ontology (GO - to standardize gene and gene product attributes across species), Molecular Interactions Ontology (PSI MI - to standardize molecular interaction and proteomics data), and Systems Biology Ontology (SBO - to standardize terms commonly used in computational modeling and systems biology). To access many ontologies in biology the Ontology Lookup Service $(\mathrm{OLS})^{1}$ provides a web service to query multiple ontologies from a single location, providing a unified output format. Often experimental data is analyzed in the context of biological ontologies, for example, by means of enrichment of ontology terms to identify statistically over-represented (inner) ontology terms.

In particular, the Gene Ontology $(\mathrm{GO})^{2}$ is an on-line database that provides a set of structured vocabularies (ontologies) for the annotation of genes, gene products and sequences. These vocabularies are used to describe the roles and properties of genes or gene products in organisms and provide a consistent characterization of gene products in various databases. Currently, there are three independent vocabularies (or parts) that are considered by the GO: molecular function, biological process, and cellular component. Biologists use such a vocabulary as a guide to answer meaningful questions, e. g., "if you were searching for new targets for antibiotics, you might want to find all the gene products that are involved in bacterial protein synthesis, but that have significantly different sequences or structures from those in humans". ${ }^{2}$ In consequence, new discoveries that change our understanding of these roles are made daily, thus making GO a dynamic data set. The GO terms are interconnected and form a directed acyclic graph (DAG). 3,4

Hierarchical clustering is a standard method to analyze and visualize large-scale experimental data in the life sciences. ${ }^{5}$ It is a statistical method for finding relatively homogeneous clusters, based on two steps: (1) computing a distance matrix containing the pair-wise distances between the biological objects (such as genes) and (2) a hierarchical clustering algorithm. Clustering algorithms can either iteratively join the two closest clusters or iteratively partition clusters starting from the complete data set. After each clustering step, the distance matrix between the new clusters and the other clusters is recalculated.

Ontologies and hierarchical clustering are widely used to support the analysis of molecular-biological data obtained by high throughput technologies. These technologies lead to an ever-increasing amount of data, which delivers a snapshot of the system under investigation and allows for the comparison of a biological system under different conditions / in different

\footnotetext{
* Corresponding author. E-mail: andreas.kerren@lnu.se
} 
developmental stages / with different genetic background. However, both, ontologies as well as hierarchical clustering, results in huge data sets of DAG- and tree-like structures. To help analyzing this data, often both views are desired: visualizing the data set (such as the expression levels of the genes in an organisms) in the context of a ontology (such as the Gene Ontology) and in the context of a clustering of the data (such as an hierarchical clustering). Although there are many solutions which deal with data that are part of two trees or hierarchies or compare two trees, to our knowledge, no solution exists for the combined visualization of an ontology (DAG) and an hierarchical clustering (tree) of one data set.

This paper proposes a new method for this task and is structured as follows: in Section 2 the properties of the input data are discussed, Section 3 presents our visualization approach combining Gene Ontology (DAG) visualization and cluster tree visualization, and discusses interaction techniques. Section 4 deals with technical issues such as implementation aspects and the scalability of the proposed method. Finally, Section 5 presents a discussion of our tool's utility as well as conclusions including ideas for future work.

\section{PROPERTIES OF THE INPUT DATA}

We use a transcriptomics data set representing different expression levels of genes. The initial data set has been reduced to genes which are significantly up- or down-regulated, resulting in 7,312 genes. The result tree of the cluster analysis (called Cluster Tree in the following) is a binary tree with 14,623 nodes and 14,622 edges. It has 7,311 (non-terminal) nodes and 7,312 leaves (terminal nodes). The Gene Ontology is a DAG consisting of more than 34,000 inner nodes and a substantial amount of leaf nodes depending on the organism under consideration. We consider only the nodes representing the 7,312 genes and those nodes, which are on paths between the GO root node and leaf nodes (genes). Therefore, the final GO data set consists of 10,042 nodes and 24,155 edges. The graph has 1 root, 2,729 (non-terminal) nodes and 7,312 other nodes. Not all of these are leaves of the GO. There is also a considerable amount of unconnected nodes as not all genes are assigned to GO terms and therefore do not form part of the GO DAG.

Both of these graphs are independent from each other from a developers point of view as they have different node and edge IDs. However, the graphs have the same label for terminal nodes (genes), indicating that they "share" a specific part of nodes among each other. This means that the relationship between these two data sets can be mapped as indicated by Figure 1. To further investigate the relations between GO DAG and Cluster Tree, users should be able to find the cluster subtree derived from any node in the GO. The main idea here is that for each interactively selected node in the Gene Ontology visualization, a corresponding subtree in the Cluster Tree should be computed. Our own implementation of this mapping (subtree calculation) is briefly described in Subsection 4.1 .

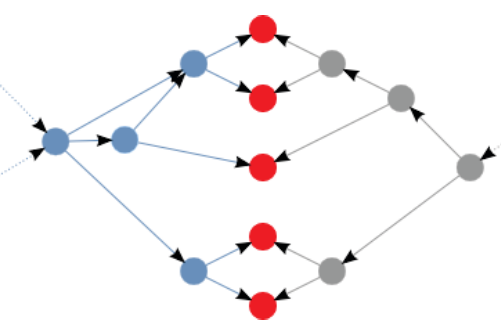

Figure 1. The light-blue part on the left represents a part of the GO DAG. The grey part on the right represents the Cluster Tree, while the red nodes in the middle are shared between both of them. Note that this diagram shows a idealized situation, because the common leaves do not need to be neighbored.

\section{VISUALIZATION APPROACH - CluMa-GO}

Due to the complexity and size of our input data, we visualize the GO DAG and the Cluster Tree in two separated and coordinated views. ${ }^{6}$ The data is fed to our tool by using two individual .gml files ${ }^{7}$ (one for the GO and one for the clustering) through a standard dialog box. Representing large data sets on their own is challenging, but our tasks became even more complicated as we have to relate two such data sets of different nature to each other: a DAG and a binary tree. We use interaction techniques such as brushing to show the mapping between both. If we draw the graphs by using conventional graph drawing algorithms, problems such as clutter when showing the GO DAG and long or wide cluster trees (depending on the chosen tree drawing algorithm) would appear. This would result in a lot of scrolling and panning actions, ${ }^{8}$ because zooming out would not be sufficient in case of the Cluster Tree visualization (traditional tree drawing 
algorithms produce much unused space). Another issue with respect to the mapping is the cluster subtree derived from a selected GO node as described in the previous section. Here, specific nodes (or complete subtrees) are not sequentially mapped and thus form "gaps", see the green leaf node between the two yellow rectangles in the background of Figure 12. These gaps might happen to be too far apart from each other to be shown in a single view. As a consequence a user might miss some information.

We implemented specific representations for the GO DAG and Cluster Tree that addresses the aforementioned challenges. First, we will present the approaches to visualize both the GO DAG and Cluster Tree and describe the supported interaction techniques later in order to distinguish between visual representations and interaction concepts. A complete overview of the GUI of our prototype implementation, called CluMa-GO, ${ }^{9}$ is shown in Figure 2.

\subsection{Gene Ontology (DAG) Visualization}

As already described in Section 2, the used GO DAG consists of more than 10,000 nodes and 24,000 edges, even if we use a subset of the entire GO. The visualization of such a graph by using standard node-link approaches would not scale without some kind of filtering or aggregation. Our challenge was to show all data in one view. We took inspiration from pixel-based approaches, which usually cope with large data sets. ${ }^{10,11}$ In our case, GO nodes are represented by colored pixels, whereas edges are hidden to avoid clutter. We call those pixels node pixels in the remainder of this paper. Choosing the right color theme was another challenge due to the use of pixel-based approaches. CluMa-GO supports an arbitrary color setting of the different elements of the visualization, such as color of the non-terminal and terminal node pixels, background, etc. In the default setting, all graphical elements can be easily distinguished and identified on a computer screen, but in order to write this paper, we found a good working compromise for both the computer display and for print outs. ColorBrewer ${ }^{12}$ turned out to be a great help for doing this.

Red node pixels represent leaf or unconnected nodes and light-blue node pixels non-terminal nodes. DAGs can be hierarchically layered and have a "flow direction" as there are no cycles. This allows us to place the nodes into several layers, which provide some insight into the topology of the GO graph as shown on the left hand side of Figure 2. This method produces results that have some similarity with the semantic substrates approach presented by Shneiderman and Aris. ${ }^{13}$ However, the placing of the nodes in layers in our approach is solely based on the graph topology, while in the semantic substrates approach, they are placed in regions (resembling our layers) based on specific node attributes. The layers are denoted by layer numbers and small line segments in the GO view to give a cue to the spatial area of the particular layers. Our GO data set has a quite big number of unconnected nodes. In this particular view, those unconnected nodes are placed in layer number 0 as shown in Figure 2 on the left. We can immediately notice that it is the most dense layer. We have implemented two layering approaches that mainly differ in the way how leaves and unconnected nodes are positioned. These approaches are discussed in the following two paragraphs.

The first layering approach is called Levels Layout and places the leaves (red node pixels) and non-terminal nodes (light-blue node pixels) into their corresponding layer depending on their graph-theoretic distance ${ }^{14}$ from the source node (root). Moreover, leaf nodes are distributed in the left part of their assigned layer; all other nodes are arranged on the right. This feature gives us further insight into the topology of a specific layer by gaining information about the distribution of leaf nodes and non-terminal nodes on a particular layer. Figure 2 shows an example of this layout strategy in the GO view on the left hand side, whereas Figure 3 displays the situation if the user zooms in the view. Although the resulting visualization looks to mimic bar charts, the number of leaves cannot be precisely compared between different layers, as the area the red node pixels (leaves) cover is not proportional to the total number of leaves in each layer. But, it is proportional to the sum of nodes in that particular layer. In other words, the covered area depends on the specific layer density. Unconnected nodes are placed in the top layer number 0 . The spatial arrangement of the node pixels within a layer, except the placing of leaves and non-terminal nodes in specific regions, is random.

Our second layering approach Bottom Layout is similar to the first one in terms of placing the nodes into corresponding layers based on the distance from the source node and random distribution of the node pixels within each layer. However, all leaves are placed into one single layer together with unconnected nodes at the bottom of the GO view, i. e., in the layer with the highest number (Figure 4). Unconnected nodes can be filtered out if necessary. This approach gives insight into the distribution of nodes among different layers without the distraction of the leaves, thus enriching the perception of the graph topology.

Edges are not shown by default in the initial view since clutter will occur otherwise. They are shown optionally in case the user selects a particular GO term (non-terminal node) for further exploration. We also implemented a simple 


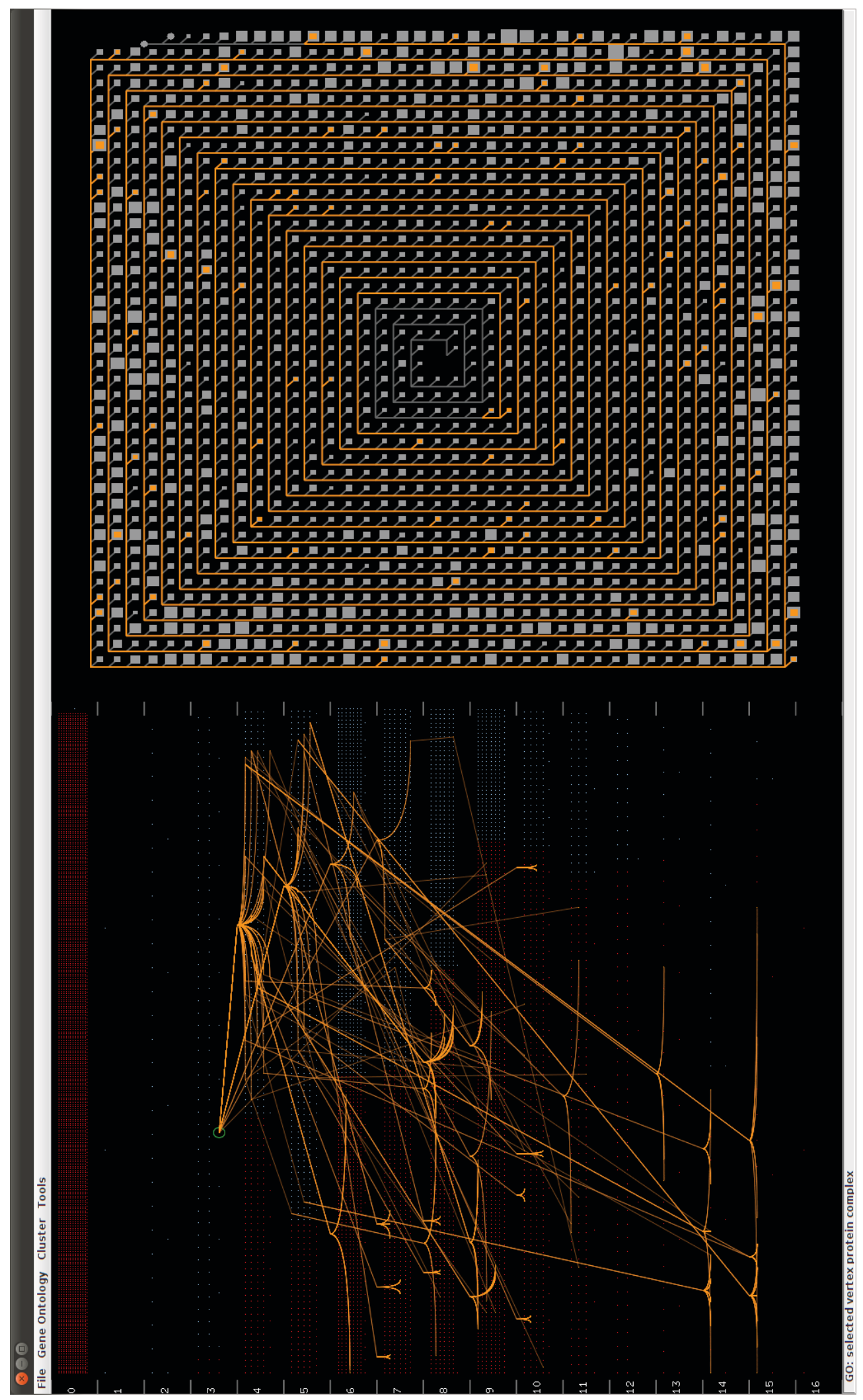

Figure 2. GUI of CluMa-GO (rotated by $90^{\circ}$ ). On the left hand side, the used Gene Ontology is represented in the GO view (Levels Layout). On the right hand side, the Cluster Tree view is located. 


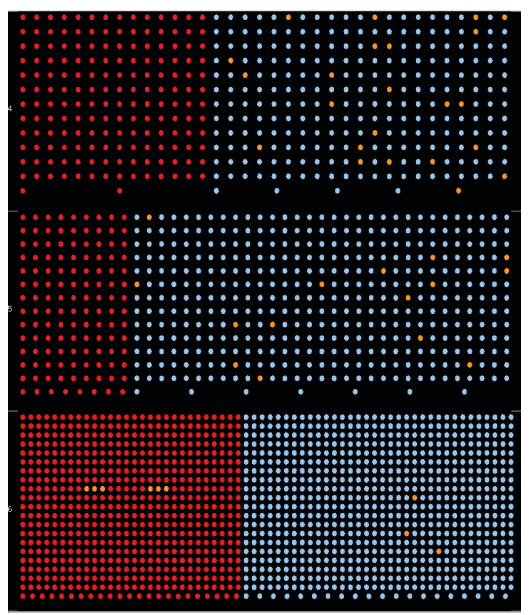

Figure 3. Zoomed-in view using the Levels Layout approach. The red nodes represent leaf nodes (e.g., genes); the light-blue nodes represent non-terminal nodes (e.g., terms). This view provides insight into the distribution of leaf nodes in a specific DAG level. The orange nodes represent the calculated subgraph (mapping).

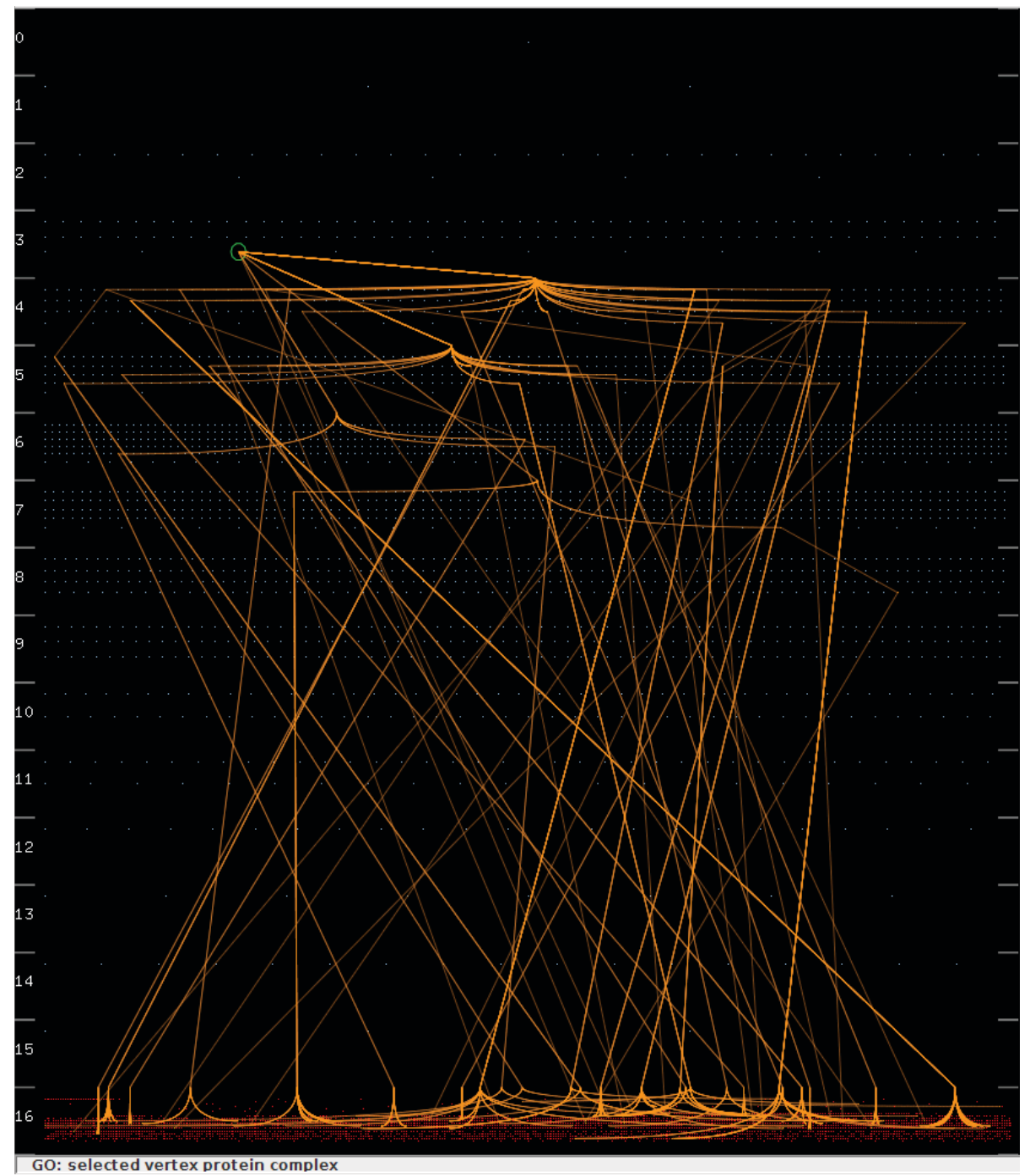

Figure 4. GO View with visible (bundled) edges based on the Bottom Layout. 
edge bundling algorithm to reduce clutter, i. e., only paths outgoing from a selected node that end up in the same layer are bundled together. Figures 2 and 4 show the edge bundling of the calculated subgraph in the GO view based on the Levels Layout and Bottom Layout approaches. This facilitates the differentiation of layers accessed by a specific node. Furthermore, placing the nodes on layers makes the use of arrows for showing the edge direction obsolete, as the flow in longest path layered DAGs is from lower layers to higher ones, i.e., from top to bottom in our case. Also, there cannot be an edge between terms in the same layer.

\subsection{Cluster Tree Visualization}

We have to address similar problems with respect to the Cluster Tree visualization as we had to do with the GO representation. The tree is usually huge and any traditional type of visualization would not scale. The application of conventional tree drawing algorithms would produce rather high tree drawings or wide ones, if we would choose to draw the entire binary tree as a dendrogram. Therefore, we developed a novel visual representation for the Cluster Tree. We have noticed that the trees in our data sets are particularly high and unbalanced with not so deep branches (subtrees) and decided to take this disadvantage of typically space-consuming drawings and turn it into an advantage when dealing with trees of such nature.

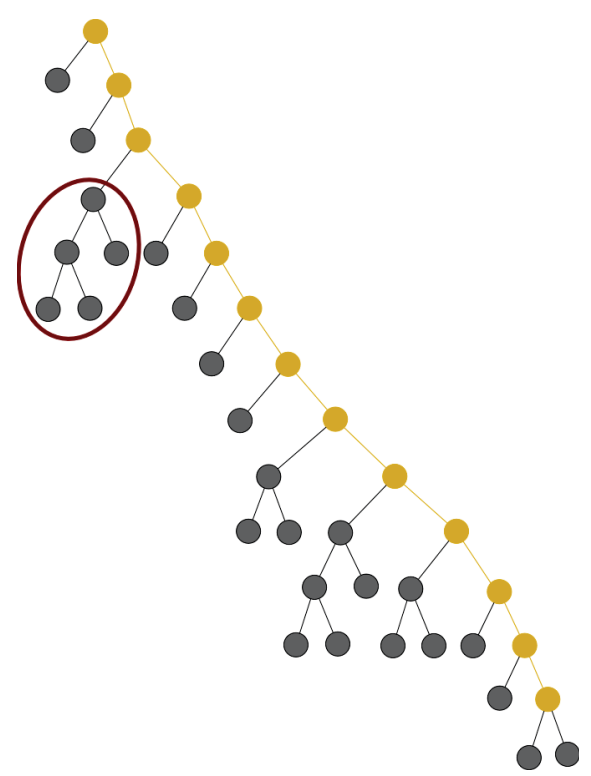

Figure 5. Sample cluster tree $t$. Yellow color represents the calculated backbone.

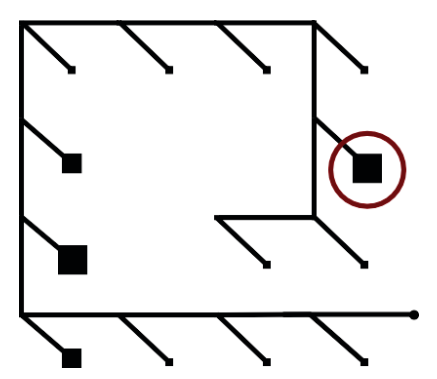

Figure 6. Spiral Tree Layout of $t$. The drawing algorithm was inspired by standard spiral layouts that are mostly used to represent timeseries, such as. ${ }^{15,16}$

Figure 5 displays how a part of such a tree might look like. We decided to use those nodes and edges that form the longest path that connects all branches as a "backbone" for our Spiral Tree Layout. We represent this backbone as a spiral, thus preserving space and giving us a possibility to show the complete tree in one view. We implemented this space-filling tree visualization approach, which deals particularly with unbalanced binary trees. This prevents us to perform repetitive scrolling to browse or navigate the elements. ${ }^{17,18}$ The direction of the flow in the spiral is counter-clockwise from the center towards out, i.e., the closer the subtrees (see below) are to the center of the spiral the closer to the Cluster Tree root they are. For instance, the sample tree $t$ in Figure 5 visualized by using our Spiral Tree Layout would look like the one shown in Figure 6.

The subtrees connected to the backbone are aggregated as the data set is too large. Thus, we allow a specific amount of abstraction in our visualization approach: each small box glyph in Figure 6 corresponds to one subtree branching out from the backbone with an angle of $135^{\circ}$ from the vertical. The size of a box glyph represents the number of nodes of the corresponding subtree. For instance, the subtree marked with the brown ellipse in Figure 5 is visualized by the box glyph marked with the brown circle in Figure 6. The highlighted subtree with five nodes is one of the largest ones; therefore, the box in the spiral is proportionally enlarged by the drawing algorithm. In the current version of CluMa-GO, the space between the "spiral arms" of the backbone is constant and not influenced by the size of the subtrees. Therefore, the box representing the subtree is normalized based on the maximum number of elements a particular subtree has. 


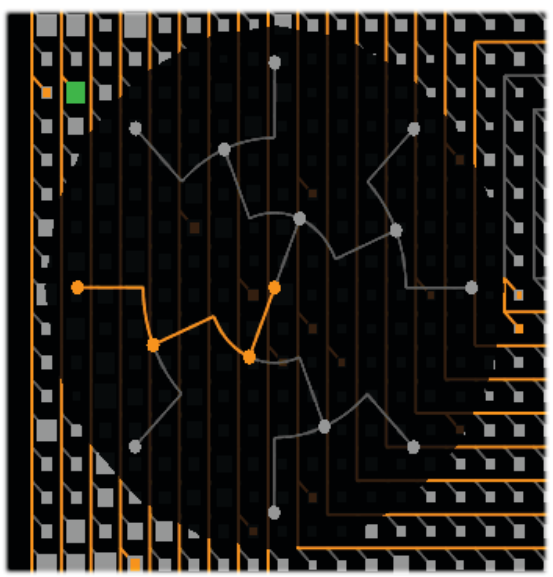

Figure 7. Subtree (branch) view. The more detailed view of the selected branch (green box glyph) is visualized as a dendrogram.

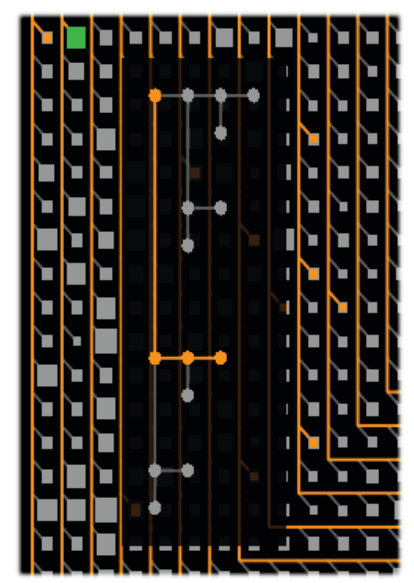

Figure 8. Subtree (branch) view. The more detailed view of the selected branch (green box glyph) is visualized by following a so-called HV-drawing algorithm.

This approach helps to identify interesting patterns of distributions of subtree branches in the Cluster Tree. For example, if we look at the Cluster Tree view in Figure 2, we notice that the biggest branches appear far away from the root node of the tree. To support a deeper analysis, the user can explore the details of each subgraph visualized in the spiral. This is done by clicking on a box glyph. CluMa-GO displays then the tree visualization widget (Figure 7 and 8) as described in Section 3.3. Here, the user has the choice between two dendrogram layouts: a radial method and a so-called HV-drawing method as well. The mapping between the two parts, GO DAG and Cluster Tree respectively, is realized by using brushing techniques. These and other interaction techniques are described in the following subsection.

\subsection{Interaction Techniques}

Biologists usually browse the data set randomly, or have a specific GO term in mind. They can either select or search for that specific term in a list that is shown in a dialog box called from the menu, or they can directly click on a particular node in the GO view. A mouse-over action on a node will display the name of that node with the help of a tool-tip. This helps the users to browse the GO and to select a node for further exploration. The GO view displays the nodes as single pixels as already explained earlier in this paper. It is pretty hard to perceive a single highlighted pixel by using color coding only. Therefore, we allow double-coding and draw a circle around the selected node in the GO view, as seen in the third layer of the GO view in Figure 4. This feature makes it also easier to identify the layer the currently selected node belongs to.

After the node has been selected by clicking, the subgraph consisting of all reachable nodes will be calculated. These related nodes, as explained in Subsection 4.1, will be highlighted in orange in the GO view. Optionally, the edges of the subgraph will be shown too. At the same time, the corresponding cluster subtree will be highlighted with the same color in the Cluster Tree view reflecting the selection made in the GO view. In this way, the user can easily identify the mapping between both views by comparing the orange colored elements. Note that the closer the selected node is to the GO root, the larger the number of nodes can be accessed from that particular node (the root node of the GO DAG, for instance, has access to all nodes of the DAG). This means that if the root node pixel is clicked, the complete DAG is selected. In such cases, clutter cannot be avoided. Therefore, users can choose the option to disable the visualization of edges if needed.

The user can also zoom in at the specific layers on the GO view (Figure 9), and scroll up or down between three layers simultaneously. The edges are not shown in the zoomed-in view, because a lot of edges from other layers might go through and in consequence introduce clutter. However, the nodes remain highlighted, and since we deal with a fixed amount of layers and magnified node pixels, it is easier to discover connections than in zoomed-out mode. The zoom-in mode is particularly helpful for analyzing different elements of the subgraph, as it is easier to select and interact with bigger node representations.

Figure 2 (right part) displays a Cluster Tree visualization with a calculated subtree highlighted in yellow, which was triggered by selection of a specific GO term. We can see that this particular GO term covers most of the backbone of the cluster tree. Some subtree box glyphs are not highlighted, while others are only partially highlighted. This is due to the 


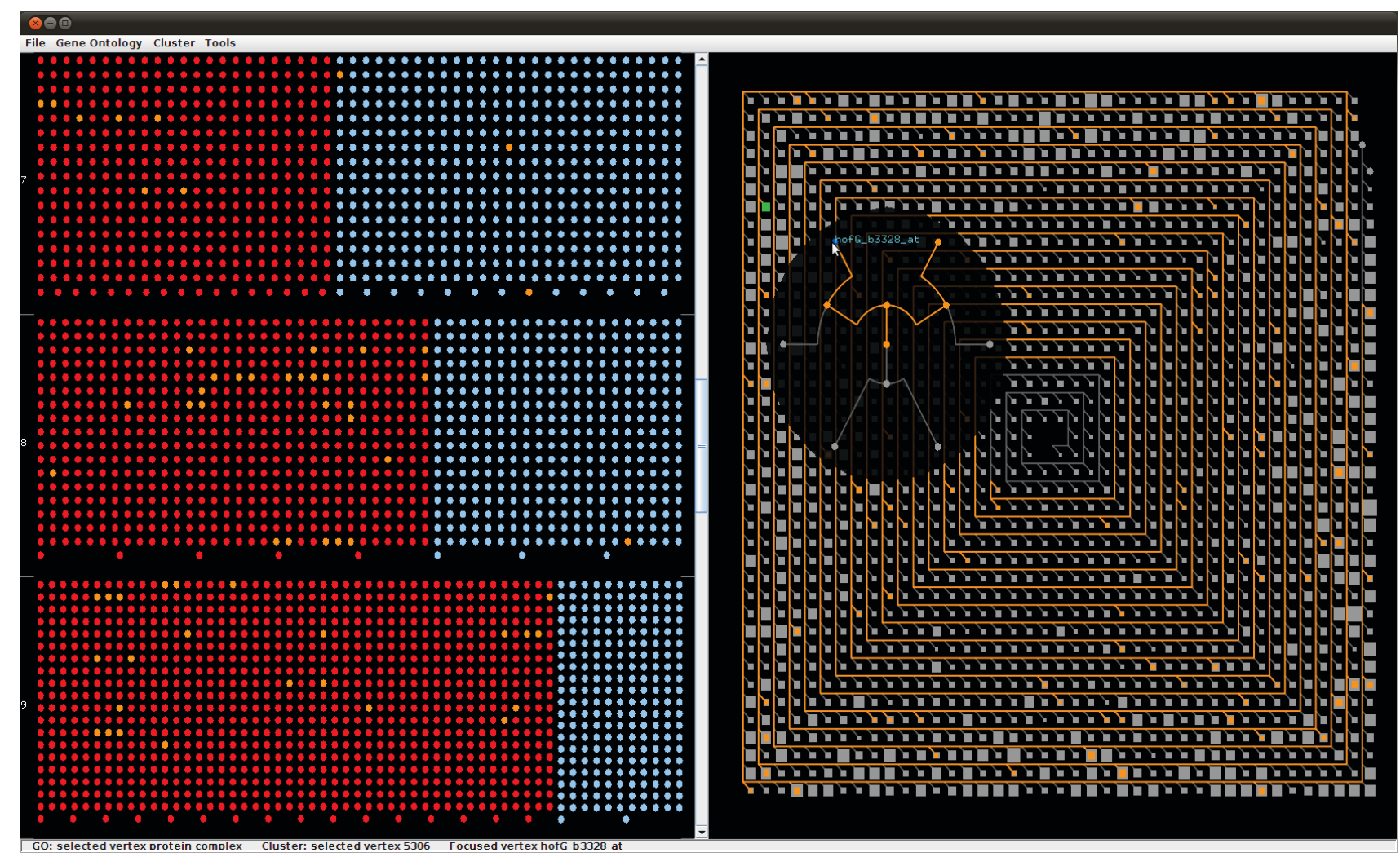

Figure 9. This screenshot shows the zoomed-in GO view on the left hand side and the Cluster Tree view with opened subtree widget on the right hand side.

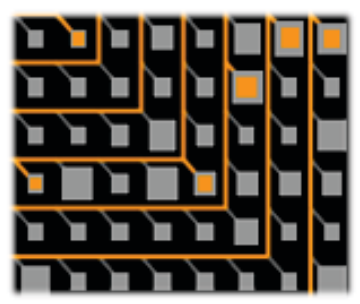

Figure 10. Cut-out of a mapping in the Cluster Tree view.

fact that not all nodes in a subtree might be mapped to the selected GO term. The area of the highlight is proportional to the number of the nodes mapped in that particular branch. Figure 10 displays a cut-out of a Cluster Tree view in order to provide a more detailed view.

Users can further examine these subtrees by clicking on them. This opens a widget that shows the particular subtree in two optional layouts that users can select based on their preference. They can view the subtree in an "explorer view" (Figure 8) based on an HV-drawing algorithm ${ }^{19}$ or as a radial dendrogram (Figure 7) similar to other dendrogram visualizations. ${ }^{20,21}$ The subtree widget appears next to the selected subtree box glyph. It is semi-transparent in order to show the context of the area that it covers. In case the area covered by the widget is important and interesting, the user can grab the widget with the mouse and move it around. Similar to the GO view, a mouse-over action shows the name of the particular node of the tree through a tool-tip.

\section{TECHNICAL ISSUES}

\subsection{Architecture and Implementation}

CluMa-GO was developed using the Java programming language and Java OpenGL (JOGL) for visualization and interaction. JOGL is a wrapper library that allows OpenGL to be used in $\mathrm{Java}^{22}$ and is the reference implementation for Java 
Bindings to OpenGL (JSR-231). To build the graphical user interface (GUI), we used the Java Swing API. It provides a native look and feel that emulates the visual appearance of several computer platforms. JOGL is just a wrapper that uses corresponding native libraries depending on the platform. Thus, builds for our tool have to be made for all popular platforms, such as Windows 32 and 64 bit versions, Mac OS X 10.6, or similar. Every build contains the necessary native libraries and Java libraries (jars).

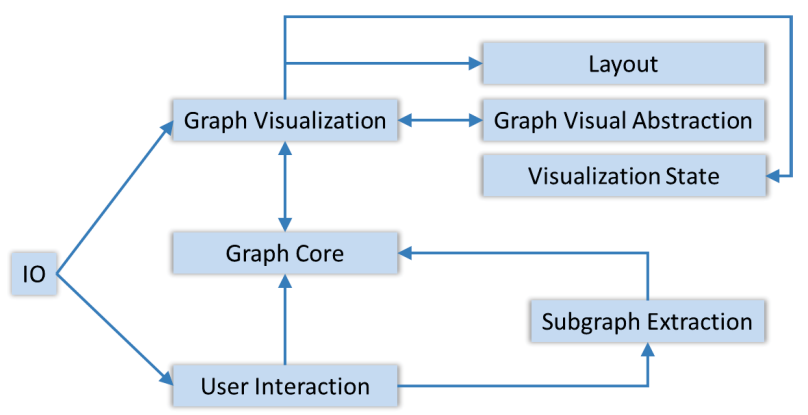

Figure 11. Module architecture of CluMa-GO.

An overview on the tool's architecture is given in Figure 11. The implementation is divided into several modules specialized for various tasks. The $\mathrm{IO}$ module implements data loading from .gml files. The data is stored in extended gml file format, which contains additional properties for nodes, such as the node label. The Graph Core module extends the JUNG graph mode ${ }^{23}$ in order to fit it to our requirements. The implementation of Swing GUI and OpenGL user interactions is realized by the User Interaction module. The Graph Visualization module and its submodules contain all code for the whole visualization process, including our own layout implementation, primitive drawing abstraction, and program state machine. One of the most important modules of CluMa-GO is of course Subgraph Extraction that contains the implementation of the subtree calculation algorithm. Figure 12 shows an instantiation of the algorithm on a given small input example. Our tool and the source code are freely available in a SourceForge repository. ${ }^{24,25}$

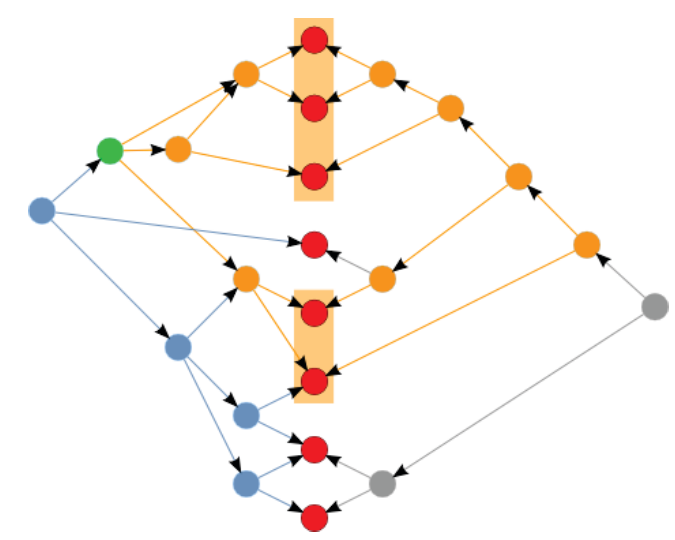

Figure 12. The red nodes in the middle are shared between both the GO DAG (light-blue nodes) and the Cluster Tree (grey nodes) (cp. Figure 1). The interactively selected node is highlighted in green, from which we traverse the graph (orange nodes) until we reach all accessible leaves (red nodes with orange background). The leaves are used to calculate a subtree of the Cluster Tree (orange nodes in the right part of the figure).

\subsection{Scalability}

When dealing with data sets as presented in Section 2, a number of issues need to be addressed. One of the main challenges is to show the complete data set to start the analysis process or to provide an overview. As explained in the previous section, we can clearly see that our prototype is able to visualize the complete data set. And with the help of the described interaction techniques, we can gain more insight into the data and perform the mapping between the GO subset and Cluster Tree.

Another issue that arises during the work with our prototype is its responsiveness. An important question is if the system can handle all data and provide the users with real-time interaction possibilities. CluMa-GO can open and visualize both 
input files in three to five seconds approximately. However, when clicking on the GO root term, the complete subgraph and subtree has to be calculated that involves the parsing of almost all nodes from both data sets. It can take up to ten seconds to be calculated on a standard PC (Core 2 Duo Intel processor with $2.53 \mathrm{GHz}$ ). This is of course the worst case scenario, and most of the nodes from the lower levels respond immediately when selected. Nevertheless, we have implemented a simple caching strategy that speeds up the process significantly (around one second to highlight the calculated subtree if the root node is chosen). Once the user has selected a particular GO term, the calculated mapping data are cached. So, the next time a user selects the same node only the highlight occurs as the calculation is stored in memory. To reduce the memory usage for caching, we used a smart map from the open source library Google Guava. ${ }^{26}$ This map allows to set a limit of stored elements together with a setting of their life times. The parameter setting in our tool currently corresponds to a storage of up to 100 subgraphs for the GO and Cluster Tree for about one minute. If one of these limits is reached then the oldest map element will be removed. Frequently used elements remain in the cache for a longer time.

\section{DISCUSSION AND CONCLUSION}

\subsection{Discussion}

Ontologies and hierarchical clustering are both important tools in biology and medicine to study high-throughput data. The presented tool supports the interaction between both analysis frameworks. A typical example is transcriptomics data. The transcriptome is the set of all RNA molecules in one or a population of cells. It is measured by DNA microarrays or sequencing and gives a snapshot of the current gene activity within the cell. Hierarchical clustering is a typical method to identify and classify patterns of gene-expression in this data. It results in an ordering of the genes such that clusters of co-expressed genes are visualized and can be used to infer gene function. Ontologies on the other hand give a functional annotation of elements; in the case of the gene ontology it gives a hierarchical annotation of gene function. The combined investigation of gene activity in both—hierarchical clustering and ontologies-can now help in better understanding the roles or functions of genes. If, for example, a small cluster of genes is highlighted in the hierarchical clustering and the visual investigation of the corresponding genes in the GO shows that most of these genes belong to the same subgroup within the ontology, then this gives a strong indication that these genes are not only assigned to the same function, but that this function may be of particular importance (as the activity of these genes behaves similarly). On the other hand, if the genes of a cluster in the hierarchical clustering belong to many different ontology concepts (assigned functions), then it may be also of interest to investigate these functions in more detail. Finally, the enrichment of ontology terms in the data is used to identify statistically overrepresented ontology terms, giving insight into relevant biological processes or functional modules. If the respective genes also behave similarly (belong to the same cluster in the hierarchical clustering), then this is again of interest to a biological user as the enrichment or clustering has been obtained independently with these two different methods. Therefore, a typical user session would be browsing the data to investigate the relation between functional annotation in the ontology and behavioral grouping of gene activity in the clustering.

\subsection{Conclusion and Future Work}

We presented a new method for the combined visualization of an ontology (represented as DAG) and an hierarchical clustering (represented as tree) of one data set. The proposed method interactively visualizes all the data without scrolling, thereby presenting an complete overview. It also allows for interactive selection and navigation to explore the data. We have showed that CluMa-GO is able to tackle the problem in our research focus, i.e., the visualization and visual mapping between two huge and conceptually different data sets. However, there are some improvements that should be performed in the future.

The current state of the prototype does not provide a way to visualize a direct mapping between a terminal GO DAG node and a cluster tree leave. A simple way to overcome this problem for one specific node is to highlight the corresponding nodes in the GO view and/or Cluster Tree view on mouse-over action. This could be easily implemented as a part of our future work.

As explained in the Section 3, the zoomed-in GO view shows three levels at the same time while displaying the subgraph by highlighting the nodes only. The edges are omitted due to clutter problems that can occur since edges from a higher level might go through the zoomed-in view to nodes in the lower layers. Since we are zoomed in, this does no make sense to show, because we have no insight from which layer those edges are coming from, nor to which layer they are going to. However, an improvement is possible by showing only edges between the three layers shown in the zoomed-in GO view. At the same time, the edge bundling algorithm could also be improved. 
We are also working on a heavily improved version of our spiral tree metaphor to cope with more balanced binary trees. One possible solution is to create something we call "nested spiral trees". The idea is to draw smaller spirals instead of aggregating larger subtrees that pass over a certain threshold of nodes into box glyphs. This approach will introduce more unused spaces, making the approach less space-filling.

\section{ACKNOWLEDGMENTS}

The authors wish to thank Christian Klukas, IPK Gatersleben, Germany, for providing the data sets used in this work as well as for his constructive comments.

\section{REFERENCES}

[1] Cote, R. G., Jones, P., Martens, L., Apweiler, R., and Hermjakob, H., "The ontology lookup service: more data and better tools for controlled vocabulary queries," Nucleic Acids Research 36, W372-W376 (2008).

[2] "Gene ontology," (last accessed: 2011-10-17). http://www. geneontology •org/.

[3] Ashburner, M., Ball, C. A., Blake, J. A., Botstein, D., Butler, H., Cherry, J. M., Davis, A. P., Dolinski, K., Dwight, S. S., Eppig, J. T., Harris, M. A., Hill, D. P., Issel-Tarver, L., Kasarskis, A., Lewis, S., Matese, J. C., Richardson, J. E., Ringwald, M., Rubin, G. M., and Sherlock, G., "Gene ontology: tool for the unification of biology," Nature Genetics 25(1), 25-29 (2000).

[4] The Gene Ontology Consortium, “The Gene Ontology project in 2008," Nucleic Acids Research 36(36), D440-D444 (2008).

[5] Eisen, M. B., Spellman, P. T., Brown, P. O., and Botstein, D., "Cluster analysis and display of genome-wide expression patterns," Proceedings of the National Academy of Sciences 95(25), 14863-14868 (1998).

[6] Roberts, J. C., "Exploratory visualization with multiple linked views," in [Exploring Geovisualization], MacEachren, A., Kraak, M.-J., and Dykes, J., eds., Elseviers (December 2004).

[7] Himsolt, M., "Gml: A portable graph file format," tech. rep., University of Passau, Germany (1997).

[8] Van Wijk, J. J. and Nuij, W. A. A., "Smooth and efficient zooming and panning," in [Proceedings of the Ninth annual IEEE conference on Information visualization ], INFOVIS'03, 15-22, IEEE Computer Society, Washington, DC, USA (2003).

[9] Kerren, A., Jusufi, I., Aleksakhin, V., and Schreiber, F., "CluMa-GO: Bring Gene Ontologies and Hierarchical Clusterings Together." Interactive Poster, IEEE Symposium on Biological Data Visualization (BioVis '11), Providence, RI, USA (2011).

[10] Keim, D. A., Ankerst, M., and Kriegel, H.-P., "Recursive pattern: A technique for visualizing very large amounts of data," in [Proceedings of the 6th conference on Visualization '95], VIS '95, 279-286, IEEE Computer Society (1995).

[11] Keim, D. A., Schneidewind, J., and Sips, M., "Scalable pixel based visual data exploration," in [Proceedings of the 1 st first visual information expert conference on Pixelization paradigm], VIEW'06, 12-24, Springer-Verlag, Berlin, Heidelberg (2007).

[12] Brewer, C. A., ColorBrewer. http: //colorbrewer2 org/, 2nd ed. (last accessed: 2011-10-17).

[13] Shneiderman, B. and Aris, A., "Network visualization by semantic substrates," IEEE Transaction on Visualization and Computer Graphics 12(5) (2006).

[14] Bondy, J. A. and Murty, U. S. R., [Graph Theory], vol. 244 of Graduate Texts in Mathematics, Springer, 3rd corrected printing ed. (2008).

[15] Aigner, W., Miksch, S., Muller, W., Schumann, H., and Tominski, C., "Visual methods for analyzing time-oriented data," IEEE Transactions on Visualization and Computer Graphics (TVCG) 14, 47 -60 (jan.-feb. 2008).

[16] Tominski, C. and Schumann, H., "Enhanced interactive spiral display," in [Proceedings of the annual SIGRAD Conference, Special Theme: Interaction], SIGRAD '08, 53-56, Linköping University Electronic Press (2008).

[17] Johnson, B. and Shneiderman, B., "Tree-maps: a space-filling approach to the visualization of hierarchical information structures," in [Proceedings of the 2nd conference on Visualization '91], VIS '91, 284-291, IEEE Computer Society Press, Los Alamitos, CA, USA (1991).

[18] Stasko, J. and Zhang, E., "Focus+context display and navigation techniques for enhancing radial, space-filling hierarchy visualizations," in [INFOVIS '00: Proc. IEEE Symposium on Information Vizualization 2000], 57, IEEE Computer Society, Washington, DC, USA (2000). 
[19] Di Battista, G., Eades, P., Tamassia, R., and Tollis, I. G., [Graph Drawing: Algorithms for the Visualization of Graphs], Prentice Hall, New Jersey (1999).

[20] Therón, R., "Hierarchical-temporal data visualization using a tree-ring metaphor," in [Smart Graphics], Butz, A., Fisher, B., Krüger, A., and Olivier, P., eds., Lecture Notes in Computer Science 4073, 70-81, Springer Berlin / Heidelberg (2006).

[21] Santamaría, R. and Therón, R., “Treevolution: visual analysis of phylogenetic trees,” Bioinformatics 25, 1970-1971 (August 2009).

[22] JogAmp, "Home of the projects JOGL, JOCL and JOAL," (last accessed: 2011-10-17). http: // jogamp . org/.

[23] O'Madadhain, J., Fisher, D., and Nelson, T., "JUNG - Java Universal Network/Graph Framework," (last accessed: 2011-10-17). http://jung. sourceforge.net/.

[24] SourceForge, "Find, create, and publish open source software for free," (last accessed: 2011-10-17). http:// sourceforge.net/.

[25] CluMa-GO, "Visualization of Mappings between the Gene Ontology and Cluster Trees," (last accessed: 2011-10-17). http://sourceforge.net/projects/go-cluster/.

[26] Google, "Guava: Google Core Libraries for Java 1.5+," (last accessed: 2011-10-17). http://code.google. $\mathrm{com} / \mathrm{p} /$ guava-libraries/. 